\title{
Estrategias metodológicas aplicadas por los docentes en la enseñanza de la Electrostática fundamentadas en el enfoque de Díaz-Barriga y Hernández
}

Methodological strategies applied by teachers in the teaching of electrostatics based on the approach of Díaz-Barriga and Hernández

$\begin{array}{lr} & \begin{array}{r}\text { Luis Torres } \\ \text { luiggi.torres94@gmail.com }\end{array} \\ \text { Recibido febrero } 2020 & \text { Ministerio del Poder Popular para la Educación, Venezuela } \\ \text { Arbitrado marzo } 2020 & \text { Código ORCID: 0000-0002-5650-7033 }\end{array}$

\section{Resumen}

El presente estudio tuvo como objetivo describir las estrategias metodológicas aplicadas por los docentes, para la enseñanza de la Electrostática, fundamentada en el enfoque de Díaz y Hernández, en el circuito № 8 del Territorio escolar № 7 del municipio Los Guayos, estado Carabobo. Fue de tipo descriptiva, con un diseño de tipo no experimental de campo, transeccional y univariable, con una población de diez docentes. Del análisis de los resultados se evidencia que las estrategias didácticas más utilizadas en la actualidad es la Postinstruccional con un $42 \%$ de los docentes encuestados que manifestaron siempre emplearlas. Sin embargo al analizar los resultados de la media se obtuvo 1,6 para la dimensión Preinstruccional, 1,25 para la Coinstruccional y 2,0 para la Postinstruccional, por lo que se ubica entre las opciones pocas veces 0 nunca con mayor tendencia a pocas veces ser utilizadas, esto de acuerdo con la escala de estimación para las respuestas del cuestionario cuyos valores fueron de (4) para la opción Siempre, (3) Casi siempre, (2) la opción Pocas veces y (1) Nunca.

\footnotetext{
Abstract

The present study aimed to describe the methodological strategies applied by teachers for the teaching of Electrostatics, based on the approach of Díaz and Hernández, in circuit No. 8 of School Territory No. 7 of the Los Guayos municipality, Carabobo state. It was descriptive, with a non-experimental field, transectional and univariate design, with a population of ten teachers. From the analysis of the results, it is evident that the didactic strategies most used at present is Post-instructional with $42 \%$ of the teachers surveyed who always said they use them. However, when analyzing the results of the mean, 1.6 was obtained for the Pre-instructional dimension, 1.25 for the Coinstructional dimension and 2.0 for the Post-instructional dimension, which is why it is among the options rarely or never with a greater tendency to few times to be used, this according to the estimation scale for the questionnaire responses whose values were (4) for the option Always, (3) Almost always, (2) the option Rarely and (1) Never.
}

Palabras clave:

Estrategias

Metodológicas; estática; enseñanza

\section{Keywords:}

Methodological Strategies; static; teaching 


\section{INTRODUCCIÓN}

L

a física es una ciencia que está en constante evolución y expansión, por ende constituye un área importante en el proceso de enseñanza y aprendizaje del sistema educativo. Sin embargo, en el contexto mundial, son notables los esfuerzos por relacionar el avance de la ciencia y la tecnología como recursos para la enseñanza, con la actualización de estrategias didácticas durante el proceso de adquisición de conocimiento en un área como esta.

En este orden de ideas, el desarrollo de las estrategias en física reviste una importancia trascendental en la educación, ya que al ritmo que va la sociedad, exige el impulso de estrategias que permitan la innovación del conocimiento y la transformación de esos medios en la búsqueda de soluciones a los diferentes contextos de la vida cotidiana que se presenten en esta área.

En Venezuela la situación no es tan diferente, pues viene relacionada con lo que ocurre en la actualidad. A pesar de los esfuerzos por parte de algunos docentes del área por motivar a sus estudiantes para que así ellos puedan adquirir el conocimiento pleno sobre el contenido, no es suficiente; ya que algunos estudiantes caen en el error de no preguntar sobre los procedimientos y ejercicios explicados en la clase, como también ocurre que algunos se enfocan más en el ejercicio y no en comprender la teoría que lo respalda porque no es relevante para ellos aprehender dicho conocimiento a través de diversos métodos que deben ser guiados por el docente sino que lo hacen memorísticamente, sin tomar en cuenta que en esta área se requiere del análisis para comprender los fenómenos.

Al respecto es necesario citar lo que dice Perrenoud (2012), el cual hace referencia a la necesidad de

una enseñanza que prepare para la vida comporta que todos los contenidos de enseñanza deben tener sentido para el alumnado, es decir, deben ser presentados desde su funcionalidad, pero centrada además en el saber hacer, o sea, el trabajo sistemático, de las habilidades, técnicas, métodos, estrategias, procedimientos, etc. (p. 14) 
Esto es debido al no aprovechamiento de ciertas estrategias principalmente motivadoras que pudiera verse como un pilar fundamental si se aplica con el fin de lograr que los estudiantes sean activos, tomando en cuenta los cambios educativos que demanda el país para tener docentes que no solo sean transmisores de información sino que vayan más allá con la intencionalidad de mejorar el rendimiento de los mismos.

En este contexto, es común observar cómo los docentes de física, planifican sus clases adaptando los contenidos a los modelos tradicionales de enseñanza, es decir, las clases se desarrollan con el docente transmisor y el alumno receptor, usando como recursos solo la pizarra y el marcador para que los estudiantes comprendan los contenidos. Tomando en cuenta estas afirmaciones Martínez (2003) sugiere que:

Un profesional de la educación debe orientarse a la búsqueda de procesos más participativos, dinamizando las actividades, visualizando nuevas formas de medir los logros académicos, dando cabida a situaciones que se orienten a la valoración del participante, que además de activar el aprendizaje permitirá el dominio de la información y una actitud crítica y creativa. (p.32)

Por consiguiente, es el docente quien juega un papel muy importante $y$, de acuerdo a sus roles debe crear las condiciones para propiciar la participación de manera activa a los estudiantes durante el proceso de enseñanza. En el caso del estudio de la Electrostática, en un aula de clase no se podría explicar si no se utilizan los recursos necesarios, por lo que el docente tiene que buscar mejores condiciones para el estudio de estos fenómenos y que los estudiantes los puedan analizar y reconozcan los elementos que influyen en el mecanismo de interacción de las cargas eléctricas.

Cabe destacar, que a la falta de estrategias y materiales que innoven la obtención de conocimientos, también se suma las condiciones en que se encuentran los laboratorios de las instituciones educativas en Venezuela, sin dejar de mencionar que muchas de ellas no cuentan con un espacio que funcione como laboratorio de física, en el cual el docente se apoyaría para 
estimular el desarrollo innovador y creativo de los estudiantes para que ellos analicen y conceptualicen los conocimientos adquiridos.

Siendo esta la problemática que se presenta en el circuito № 08 del Territorio escolar № 7 del municipio Los Guayos, estado Carabobo, donde los docentes no se escapan de esta realidad ya que de acuerdo a un recorrido realizado por las instituciones que conforman el circuito se pudo constatar que la enseñanza de la física básicamente se realiza de una forma teórica, donde el aprendizaje es memorístico y el docente se apoya en el uso casi exclusivo del libro para dictar la clase.

Por estas razones es que se hace necesaria la innovación en las estrategias de enseñanza para la explicación detallada de los fenómenos electrostáticos durante los momentos didácticos de la clase que, a pesar de que se puede explicar mediante ejemplificaciones de la vida diaria, estas situaciones tomadas como ejemplos requieren del estudio tanto de las características y los elementos que influyen en este fenómeno como de la comprensión de los conceptos, apoyándose de otros mecanismos como medios estratégicos para una mejor comprensión y así enriquecer el proceso de enseñanza y aprendizaje, que no sea tradicional, sino que sea una presentación que facilite el análisis.

Ante estos planteamientos surge la siguiente interrogante: ¿Cuáles son las estrategias utilizadas por los docentes de física para la enseñanza del contenido de Electrostática en el circuito № 08 del Territorio escolar № 07 del municipio Los Guayos? Para dar respuesta a la pregunta objeto de investigación se planteó el siguiente objetivo general: Describir las estrategias metodológicas aplicadas por los docentes, para la enseñanza de la Electrostática, fundamentada en el enfoque de Díaz y Hernández, en el circuito № 8 del Territorio escolar № 7 del municipio Los Guayos.

El objetivo general se desgloso en los siguientes objetivos específicos para poder darle respuesta dentro de la investigación: 1. Indicar las estrategias metodológicas preinstruccionles aplicadas por los docentes de física en la enseñanza de la Electrostática. 2. Determinar las estrategias metodológicas coinstruccionales utilizadas por los docentes de física en la enseñanza de la Electrostática. 3. Precisar las estrategias metodológicas postinstruccionales aplicadas por los docentes de física para la enseñanza de la Electrostática en el circuito № 8 del territorio escolar № 7 del municipio Los Guayos. 
Al respecto, Tamayo y Tamayo (1992) señala que en la sección

antecedentes se trata de hacer una síntesis conceptual de las investigaciones o trabajos realizados sobre el problema formulado con el fin de determinar el enfoque metodológico de la misma investigación. El antecedente puede indicar conclusiones existentes en torno al problema planteado. (p.73)

A continuación se presentan las investigaciones afines con este estudio, cuyos hallazgos permiten relacionar y profundizar la problemática.

En el trabajo realizado por Muñoz y Vélez (2018), denominado "Estrategias metodológicas que utilizan los docentes para la enseñanza de la Ley de Ampere bajo el enfoque de Díaz y Hernández", los autores describen las estrategias metodológicas que utilizan los docentes al enseñar la ley de Ampere, haciendo referencia a las estrategias docentes de Díaz y Hernández (2002) y a la teoría del aprendizaje significativo del psicólogo Ausubel (2006).

Los datos obtenidos en la investigación dieron como resultado que casi todos los docentes del área de Física dan un inicio adecuado a la clase trabajando con los conocimientos previos que ya tiene el estudiante, el desarrollo de la clase basado en ilustraciones y esquemas y por último, el cierre de la clase donde si sintetizan la información. Los autores del mencionado trabajo, llegaron a la conclusión de que hoy en día existen una serie de estrategias metodológicas que los docentes pueden implementar para hacer llegar los contenidos a los estudiantes de manera efectiva. Sin embargo, no siempre se implementan de manera adecuada.

Por otro lado, Hernández y Torres (2017), en su trabajo titulado "Estrategias lúdicas para la Enseñanza del Electromagnetismo en Bachillerato", proponen un conjunto de estrategias que las autoras consideran lúdicas, para la comprensión del electromagnetismo. Las estrategias descritas presentan rasgos de juego dada la actividad competitiva que se desarrolla en grupos, en donde se les solicita a los estudiantes la realización de tareas que pongan en práctica los conceptos electromagnéticos abordados inicialmente, para después reproducirlos a través del juego y la competencia. Los resultados y conclusiones de este trabajo de investigación, arrojan 
que toda la población estudiantil se notó interesada y motivada al desarrollar y construir dispositivos como bocinas, cañones y trenes electromagnéticos en base a los contenidos vistos en clase, y que en este caso resultaban ser una réplica de los dispositivos que se usan cotidianamente.

Otro trabajo consultado fue el de López (2016), titulado "Estrategias metodológicas para mejorar el Aprendizaje del Electromagnetismo a través del uso de experimentos demostrativos", el cual llevó a cabo una propuesta basada en la realización de un conjunto de experimentos que tuvieran la capacidad de demostrar los fenómenos electromagnéticos presentes en algunos de los contenidos de física, con el fin de mejorar el aprendizaje y la promoción de actividades por parte de los educandos. Cabe señalar que estas estrategias cuya técnica es la experimentación, además tiene por objeto, suplir la carencia de experiencias de laboratorio muchas veces reflejadas en los espacios académicos destinados para esta actividad. Las conclusiones de esta investigación señalan que los docentes en su mayoría, no implementan todas las estrategias metodológicas que se requieren para la enseñanza del electromagnetismo y, a su vez, se observó que pocos ejecutan actividades de índole teórico práctico.

En el mismo orden de ideas, Colmenares y Henríquez (2011), en su trabajo especial de grado titulado "Estrategias metodológicas utilizadas por los docentes para la Enseñanza del contenido Leyes de Newton, basada en el enfoque constructivista de Díaz y Hernández en el Liceo Bolivariano Manuel A. Malpica del municipio Naguanagua del estado Carabobo", llevaron a cabo un análisis sobre un conjunto de estrategias didácticas que pudieran ser implementadas para la enseñanza del contenido de las Leyes de Newton, enmarcada en las estrategias de enseñanza que sugieren Díaz y Hernández (2002), con el objeto de lograr una mejor apropiación por parte de los estudiantes hacia los contenidos relativos a estas leyes. Las conclusiones de este trabajo de investigación arrojaron resultados positivos en el uso de estas estrategias docentes, permitiéndole a la población estudiantil apropiarse de estos contenidos a través del uso de estrategias clasificadas por dimensiones.

Todos los autores acá citados, convergen en señalar la necesidad de implementar estrategias didácticas efectivas, que motiven a los estudiantes para obtener resultados positivos en 
cuanto al logro de un aprendizaje significativo, tomando en consideración que los docentes deben crear las condiciones y reunir ciertas características adaptadas a cada realidad educativa para la aplicación de estrategias que den paso a que el estudiante pueda relacionar lo aprendido previamente con lo que se quiere obtener durante y después del momento didáctico de enseñanza y aprendizaje.

De acuerdo a Tamayo y Tamayo (1992) define teoría como "un conjunto de proposiciones lógicamente articuladas que tiene como fin la explicación y predicción de las conductas en un área determinada de fenómenos de estudio" (p.71). En este sentido, el autor sostiene que a través de las bases teóricas es que se explica lo que determina la finalidad de los fenómenos que son objetos de estudio, ya que el desarrollo de las bases teóricas que concierne al problema de investigación planteado, se basa en la recopilación de información importante y necesaria que sirva de soporte al estudio y de guía al investigador.

De acuerdo a la estructura social, para llevar a cabo todas estas propuestas se hace necesario que el docente, en el desarrollo de las potencialidades de sus estudiantes, tome en consideración cuatro pilares fundamentales según Unesco (1996) donde señala:

Aprender a conocer: el cual hace énfasis en los métodos que debe utilizar para conocer porque no todos los métodos que se usan sirven para aprender a conocer, y asegura que, en el fondo, debe existir el placer de comprender y descubrir.

Aprender a hacer: con el fin de adquirir las competencias que capaciten al individuo para hacer frente a las situaciones que se presenten y así trabajar en equipo para hacer cosas que aporten algo a la sociedad, a través de la formación para hacer un trabajo.

Aprender a convivir: invita a descubrir progresivamente al otro para observar que existen diferencias con respecto al otro. Y para descubrir al otro, se debe conocer a sí mismo para poder entender que el otro tiene sus razones y piensa diferente.

Aprender a ser: señala a la educación integral como el desarrollo total y máximo posible de cada persona, considerando al ser con pensamiento autónomo.

En este sentido, la educación venezolana va más allá del estilo tradicional entre la educación secundaria y permanente que coincide con una noción formulada a menudo: la sociedad educativa en la que todo puede ser ocasión para aprender y desarrollar las 
capacidades del individuo. Se debe concebir la educación como un todo, a partir de allí buscar la inspiración y orientación de las reformas educativas, en la elaboración de los programas y definición de nuevas políticas educativas.

El proceso de cambio del diseño curricular de educación media general que se lleva a cabo en Venezuela, señala que más que los planes de estudio y los programas de las unidades curriculares, cuando se habla de cambio curricular están involucrados los propósitos, conceptos y enfoques de la educación, las maneras de entender y valorar las prácticas educativas, los materiales y recursos para los aprendizajes.

Es decir, que mediante este proceso de adecuación se debe tomar en cuenta todas las formas posibles de enseñanza y aprendizaje, valorando y entendiendo el uso de prácticas educativas que como bien lo dice, estén enmarcadas en la necesidad de enseñar mediante nuevas estrategias. Dichas prácticas educativas nos exigen una revisión de conceptos y maneras de hacer que apunten a un quehacer educativo caracterizado por la comprensión crítica y en profundidad del mundo en que vivimos por las múltiples posibilidades de expresión creativa y exploración de las potencialidades personales del educando.

Por consiguiente, este proceso requiere de sistematización y evaluación permanente para mejorar la práctica educativa y reorientarla en cada paso del camino en función del logro de los pilares de la educación. Y en efecto, destaca que el desarrollo de las potencialidades humanas, tal es el caso de los docentes y los estudiantes; es un proceso abierto, que no tiene límites, refiriéndose a las capacidades para vivir, pensar, sentir, percibir, actuar, transformar, disfrutar, crear, construir que tienen cada uno. Lo que supone entonces la creación de un conjunto de experiencias, aprendizajes, acciones, situaciones educativas que permitan el desarrollo de estas potencialidades.

Dentro de este orden de ideas, es importante señalar que el sistema educativo del país maneja el proceso de enseñanza y aprendizaje mediante los Referentes éticos y procesos indispensables que plantean la formación integral de los estudiantes, donde se recoge la necesidad de utilizar estrategias dirigidas hacia el descubrimiento y la creación de espacios para la innovación. 
En lo referente al aspecto psicológico, conviene resaltar que en un trabajo de investigación es necesario tomar en cuenta el desarrollo de teorías que lo sustenten en este aspecto. En este orden de ideas, se puede citar a Ausubel (2001) y su teoría del aprendizaje significativo, quien expuso que:

La esencia del proceso del aprendizaje significativo reside en que las ideas expresadas simbólicamente son relacionadas de modo sustancial y no arbitrario (no al pie de la letra) con lo que el alumno ya sabe, señalando algún aspecto esencial de su estructura de conocimientos (por ejemplo, una imagen, un símbolo ya con significado, un contexto o una proposición). (p. 26)

Según el autor el aprendizaje significativo parte de lo que el estudiante ya sabe, pues relaciona nuevas ideas e informaciones que pueden ser aprendidas y retenidas siempre y cuando la conceptualización sea la adecuada y se encuentre clara y disponible en la estructura cognitiva del individuo y surja a través de la transformación de esos conceptos nuevas ideas como base para su aprendizaje. Cuando esas nuevas ideas toman un significado para el individuo por la interacción con la información que ya posee, el aprendizaje es significativo ya que el docente debe motivarlo para adquirir o relacionar conocimientos que despierten curiosidad e interés en el contenido para poder generar nuevos conocimientos.

Otro de los aspectos resaltantes en esta teoría del aprendizaje es la memorización de la información, lo que contrasta con este trabajo de investigación ya que ciertamente una estrategia de enseñanza no debería ir en este enfoque sino que debe permitir que el estudiante utilice la información que posee y la relacione con lo que va a aprender mediante la incorporación de otros métodos en su proceso de aprendizaje.

Asimismo, Ausubel (2001) habla del significado del aprendizaje, es decir la forma en que el estudiante adquiere el conocimiento o lo toma para sí, y lo escribe textualmente así:

Independientemente de cuánto significado potencial sea inherente a la proposición especial, si la intención del alumno consiste en memorizar arbitraria y literalmente (como una serie de palabras relacionadas caprichosamente), tanto el proceso de aprendizaje como los resultados del mismo serán mecánicos y 
carentes de significado. $\mathrm{Y}$, a la inversa, sin importar lo significativo que sea la actitud del alumno, ni el proceso ni el resultado del aprendizaje serán posiblemente significativos si la tarea de aprendizaje no lo es (s.n.)

Es por ello que se considera necesario la adquisición del conocimiento de manera sustancial mediante el proceso de enseñanza de la física, ya que al ser una ciencia natural requiere de estrategias que permitan al educando adquirir un aprendizaje significativo, en el cual los procedimientos para el cálculo de ejercicios que se utilizan en el caso del contenido de la Electrostática no obliguen al estudiante a aprenderse esos procedimientos matemáticos memorística y repetitivamente. Por lo que es necesario que el estudiante busque por sus propios medios y con el apoyo del docente, la forma de nutrir más su conocimiento y la capacidad cognitiva que lo lleve a relacionar lo que ya conoce con las nuevas ideas que se le plantean.

En este sentido, en su trabajo, Ausubel (2001) plantea tres tipos de aprendizaje significativo. Los cuales son: el aprendizaje por representaciones, por proposiciones y por conceptos.

Casi siempre es muy conveniente saber representar el nuevo concepto aprendido con una sola palabra de significado equivalente a éste. Pero aprender lo que significa el concepto mismo, que en efecto consiste en aprender cuáles son sus atributos de criterio, implica un tipo muy diferente de aprendizaje significativo que, como el de proposiciones, es de naturaleza e intención sustantiva en lugar de nominalista o representativa.

\section{MÉTODO}

1 l marco metodológico se refiere a las vías a seguir desde que se inicia la investigación hasta la finalización de la misma.

Por consiguiente, Balestrini (1998) define que el fin del marco metodológico es:

Situar en el lenguaje de investigación los métodos e instrumentos que se emplearan en el trabajo planteado, desde la ubicación acerca del tipo de estudio y el diseño 
de investigación, su universo o población, su muestra, los instrumentos y técnicas de recolección de datos, la medición, hasta la codificación, análisis u presentación de los datos. (p.114)

Según Arias (2004) la investigación descriptiva consiste en "la caracterización de un hecho, fenómeno o grupo con el fin de establecer su estructura o comportamiento. Los resultados de este tipo de investigación se ubican en un nivel intermedio en cuanto a la profundidad de los conocimientos se refiere" (p.48).

En atención a la definición anterior, el trabajo se ubicó en el nivel descriptivo, puesto que se procedió a recopilar toda la información relativa a las estrategias metodológicas aplicadas por los docentes para la enseñanza de la Electrostática, para caracterizarla y establecer el comportamiento de sus principales indicadores. Dicha información se analizó posteriormente para determinar los rasgos específicos que la caracterizan.

La presente investigación se basó en un diseño de campo, ya que permitió analizar y registrar datos obtenidos de la realidad de manera directa, donde ocurren los hechos, sin manipular o controlar variable alguna. En el manual UPEL (2003) se define a la investigación de campo como:

El análisis sistemático de problemas en la realidad con el propósito bien sea de describirlos, interpretarlos, entender su naturaleza y factores constituyentes, explicar sus causas y efectos o predecir su ocurrencia, haciendo uso de métodos característicos de cualquiera de los paradigmas o enfoques de investigación conocidos o en desarrollo. Los datos de interés son recogidos en forma directa de la realidad; en este sentido se trata de investigaciones a partir de datos originales o primarios. (p.14)

Asimismo dicha investigación está enmarcada en un diseño no experimental, ya que la misma se realiza sin manipular deliberadamente las variables, es decir se observa el fenómeno tal y como se da en su contexto natural, para analizarlos después.

Según lo establecen Hernández, Fernández y Baptista (2006): 
La investigación no experimental es cualquier investigación en la que resulta imposible manipular o asignar aleatoriamente a los sujetos 0 a las condiciones. De hecho, no hay condiciones o estímulos a los cuales se expongan los sujetos del estudio. Los sujetos son observados en su ambiente natural, en su realidad. (p. 289)

A su vez, la investigación se fundamenta en un diseño transeccional, puesto que se recolectan datos en un solo momento, en un tiempo único, el cual se denomina univariable. Su propósito es describir variables y analizar su incidencia e interrelación en un momento dado, así como también busca la incidencia y los valores en que se manifiesta una o más variables.

En conclusión este trabajo se trata de una investigación de tipo descriptiva, con un diseño no experimental de campo, transeccional y univariable.

Para Balestrini (1998) se entiende por población “cualquier conjunto de elementos de los que se quiere conocer o investigar, alguna o algunas de sus características" (p.122). Para la presente investigación, la población estuvo conformada por diez (10) docentes de la asignatura de Física pertenecientes al circuito № 08 del Territorio escolar № 07 del municipio Los Guayos del estado Carabobo. Son cuatro liceos: uno público y tres privados: U.E. Egidio Montesinos, U.E. Antonio José de Sucre, U.E. Pólita De Lima y U.E. Batalla de Junín.

En vista de que la población es finita, y no tratándose estrictamente de un censo poblacional, no se requiere para este estudio muestra ni muestreo. Así lo refiere Arias (2004) al manifestar que si la población en estudio resulta accesible en su totalidad, no se hace necesario la toma de muestras. Los datos se toman directamente de la población objetivo.

En esta investigación, por ser descriptiva con diseño de campo, se utilizó la técnica de la encuesta la cual definida por Hurtado (2012) "corresponde a un ejercicio de búsqueda de información acerca del evento de estudio, mediante preguntas directas, a varias unidades, o fuentes..." (p.875).

Cabe destacar que el instrumento de recolección de datos es, en principio cualquier recurso del que se vale el investigador para acercarse a los fenómenos y extraer de ellos información, por lo tanto Hurtado (2012) afirma que "un instrumento de recolección de 
datos representa la herramienta con la cual se va recoger, filtrar y codificar la información, es decir, el con qué" (p.161).

Por consiguiente, para la recolección de información el instrumento utilizado fue el cuestionario, definido por Hurtado (2012) "como un conjunto de preguntas relacionadas con el evento de estudio". El instrumento fue elaborado por Colmenares y Henríquez (2011), para la investigación Estrategias metodológicas utilizadas por los docentes para la enseñanza del contenido Leyes de Newton, basada en el enfoque constructivista de Díaz y Hernández en el Liceo Bolivariano "Manuel A. Malpica".

Dicho instrumento consta de diecinueve (19) preguntas, estas a su vez ordenadas según los momentos de enseñanza enfocados en Díaz y Hernández, los cuales son el Preinstruccional, el Coinstruccional y el Postinstruccional, basado a su vez en la escala Likert, por ser un instrumento de respuesta policotómicas se les dio un valor entre uno (1) y cuatro (4), quedando asignado a cada respuesta un valor como: Siempre (4), Casi siempre (3), Pocas veces (2), Nunca (1).

La validez se define según Hernández, Fernández y Baptista (2006), como "El grado en que un instrumento realmente mide las variables que pretende medir" (p.243). De este modo se puede apreciar que el instrumento de recolección de datos debe contar con varios elementos que evaluados bajo parámetros específicos arrojen que este tiene coherencia con las variables de estudio y pertinencia con los objetivos de la investigación. La validez del cuestionario no se realizó en este trabajo, debido a que el mismo fue validado por los investigadores (autores) Colmenares y Henríquez (2011).

En lo que concierne a la confiabilidad según Fernández, Hernández y Baptista (2006) se refiere "al grado en que su aplicación repetida al mismo sujeto y objeto, produce iguales resultados," (p.242). La confiabilidad del instrumento fue determinada por primera vez por Colmenares y Henríquez (2011) usando el coeficiente de Alfa de Cronbach arrojando un valor de 0.81 de fiabilidad, que de acuerdo a Hernández, Fernández y Baptista (2006):

Requiere una sola administración del instrumento de medición y produce valores que oscilan entre 0 y 1 . Su ventaja reside en que no es necesario dividir en dos mitades a los ítems del instrumento de medición, 
simplemente se aplica la medición y se calcula el coeficiente. (p. 354)

Sin embargo, este no pudo ser calculado para la presente investigación debido a lo pequeño de la población. En total, diez (10) docentes de Física representan la población objeto de estudio.

El procedimiento metodológico es donde se describen las fases que se cumplieron en el desarrollo de la investigación. Según Arias (2004), el proceso metodológico "es el camino que sigue la ciencia para alcanzar sus objetivos" (p. 127). Por lo tanto, es el camino que debe seguir el investigador para llevar a cabo el trabajo. El mismo comprendió los siguientes procedimientos:

Elección del instrumento: Se escogió un instrumento de 19 preguntas en base al contenido de Electrostática y a los momentos de enseñanza del enfoque constructivista de Díaz y Hernández, dicho instrumento fue elaborado en forma de cuestionario de preguntas cerradas, ya que se establecieron las opciones de respuestas que puede elegir el encuestado. Aplicación del instrumento a los docentes: Una vez obtenidos los resultados de la confiabilidad y verificar que el instrumento era confiable se procedió a aplicar el instrumento al grupo integrado por 10 docentes, el cual, una vez aplicado se recolectaron los datos correspondientes para realizar los cálculos y así obtener resultados de la investigación.

Codificación, tabulación de los datos, análisis e interpretaciones de los resultados: Con la aplicación del cuestionario se obtuvieron resultados con los cuales se procedió a tabular a través de la estadística descriptiva y así analizar cada uno de ellos para establecer conclusiones y recomendaciones.

Según Méndez (2001):

el análisis de los resultados como proceso implica el manejo de los datos obtenidos y contenidos en cuadros, gráficos y tablas. Una vez dispuestos, se inicia su comprensión teniendo como único referente el marco teórico sobre el cual el analista construye conocimiento sobre el objeto investigado. (p. 220)

Una vez aplicado el instrumento a los diez (10) docentes de Física del Circuito № 08 del Territorio escolar № 07 del municipio Los Guayos, se procedió a recolectar los datos para los cuales se 
elaboró tablas de resultados para redactar las respuestas de los docentes, así mismo se tabularon los datos aplicando la estadística descriptiva donde se puede evidenciar los datos precisos, los cuales se utilizaran en las tablas y gráficos para emitir un análisis interpretativo de los resultados de cada uno.

\section{RESULTADOS Y DISCUSIÓN}

$\mathrm{E}$ n primer lugar, al analizar los resultados obtenidos con la aplicación del cuestionario a los docentes de física del Circuito № 08 del Territorio escolar Los Guayos № 07, se pudo evidenciar que en su mayoría no emplean las estrategias metodológicas de enseñanza fundamentadas en el enfoque de los pedagogos Díaz y Hernández, durante los tres momentos didácticos de la clase: inicio, desarrollo y cierre; ya que como se pudo observar un grupo mayoritario de ellos pocas veces o nunca las han trabajado en el aula.

Con respecto a las Dimensiones en que se basa la investigación, las cuales son Preinstruccional, Coinstruccional y Postinstruccional, se pudo evidenciar, de acuerdo a los datos recabados y al hacer un análisis sobre las opciones Casi siempre, Pocas veces y Nunca se logró determinar que en conjunto acumulan la mayor frecuencia y por ende los docentes de física del Circuito donde se realizó el estudio, no toman en cuenta ninguna de las estrategias didácticas basadas bajo este enfoque metodológico que plantean los autores antes mencionados, esto, de acuerdo a lo arrojado al estudiar la media por cada dimensión, la cual se ubicó entre los valores 1 y 2 para las tres dimensiones, ubicándose de acuerdo a la escala de valoración para las opciones del cuestionario con mayor tendencia hacia la opción pocas veces.

Las influencias adversas, señaladas anteriormente, son consecuencia de la ausencia de información que tienen los docentes para que generen una visualización integral compartida en torno a las nuevas visiones, objetivos y metas que se está planteando, a pesar de los esfuerzos realizados por parte de algunos de física de contribuir a través de la enseñanza del contenido de electrostática utilizando estrategias que favorecen el aprendizaje de los estudiantes.

Finalmente, se encontró que si dichos indicadores no son ejecutados en la fase Preinstruccional, es decir, en el inicio de la 
clase hay gran incidencia de que pocas veces o nunca se apliquen durante el desarrollo o el cierre de la clase, como lo dejaron saber de acuerdo a sus respuestas los educadores del Circuito en cuestión.

\section{CONCLUSIONES}

$\mathrm{E}$ l desarrollo de este trabajo evidenció las pocas veces que son utilizadas las estrategias didácticas propuestas por los pedagogos Díaz y Hernández en el circuito № 8 del Territorio escolar Los Guayos, objeto de estudio. A continuación se enuncian un conjunto de sugerencias para influenciar a los docentes de dicho circuito hacia la aplicación de estrategias que promuevan el aprendizaje significativo.

Mejorar los procesos de comunicación que permitan lograr una visión integral compartida entre todos los docentes del área de física del circuito y hablar de la propuesta didáctica de los pedagogos antes mencionados en los llamados Consejos circuitales, facilitaría que el docente se integre y maneje los conocimientos al respecto y así lo pueda trabajar en las aulas de clases.

Ahora bien, a nivel institucional se propone afianzar el compromiso y motivación al logro, para que los docentes del área de física de dicho circuito, mantengan un estímulo renovador que les permita trabajar en función de algo, que es lograr el aprendizaje significativo de los estudiantes a través del empleo de estrategias didácticas basadas en el inicio, desarrollo y cierre de la clase de física como lo son: las estrategias didácticas Preinstruccionales, Coinstruccionales y Postinstruccionales.

Planificar las clases de acuerdo a las estrategias anteriormente mencionadas, para la enseñanza y el aprendizaje, en sus tres momentos de manera que el estudiante mejore la atención y la vez organice, codifique y sintetice la información principal.

Fomentar la participación, implementando actividades que motiven a los estudiantes y se hagan participantes del proceso de enseñanza, de tal forma que se flexibilice en cualquiera de sus tres momentos. 


\section{REFERENCIAS}

Arias, F. (2004). El Proyecto de investigación. Guía para su elaboración. Caracas: Episteme

Ausubel, D. (2001). Psicología educativa. Un punto de vista cognitivo. México: Trillas

Balestrini, M. (1998). Cómo se elabora el proyecto de investigación. Caracas: Consultores Asociados BL Servicio Editorial Briceño

Colmenares, J. y Henríquez, L. (2011). Estrategias metodológicas utilizadas por los docentes para la Enseñanza del contenido Leyes de Newton, basada en el enfoque constructivista de Díaz y Hernández en el Liceo Bolivariano Manuel A. Malpica del municipio Naguanagua del estado Carabobo. Trabajo de grado no publicado. Universidad de Carabobo, Carabobo, Venezuela

Díaz-Barriga, F. Y Hernández, G. (2002). Estrategias docentes para un aprendizaje significativo, Una interpretación constructivista. (2 ${ }^{\mathrm{a}}$ Ed.). México: McGraw-Hill

Hernández, R., Fernández, C. y Baptista, M. (2006). Metodología de la Investigación. ( $4^{\circ}$ Ed.). México D.F.: McGraw-Hill

Hurtado, J. (2012). El Proyecto de Investigación. Comprensión Holística de la Metodología y la Investigación. Sypal, Caracas, Venezuela

Ley Orgánica de Educación. (2009). Gaceta Oficial de la República Bolivariana de Venezuela, 5.929 (Extraordinario) agosto 15, 2009

Martínez, N. (2003). La importancia de promover en el aula Estrategias de aprendizaje para elevar el Nivel Académico en los estudiantes. España. Universidad de Almería

Méndez, I. (2001). El protocolo de Investigación. México: Trillas

Muñoz, Y. y Vélez, E. (2018). Estrategias metodológicas que utilizan los docentes para la enseñanza de la Ley de Ampere bajo el enfoque de Díaz y Hernández. Trabajo de Grado no publicado. Universidad de Carabobo, Carabobo, Venezuela

Ministerio del Poder Popular para la Educación. (2015). Proceso de Cambio Curricular en Educación media. Caracas, Venezuela

Perrenoud, P. (2012). Cuando la escuela pretende preparar para la vida. ( $1^{\circ}$ Ed.). Barcelona: Editorial GRAÓ

Piaget, J. (1969). La Enseñanza de las matemáticas Modernas. Madrid: Alianza Universidad

Sabino, C. (1986). Metodología de Investigación. México: Diana

Tamayo, y Tamayo, M. (1992). El proceso de la Investigación científica. ( $2^{\mathrm{a}}$ Ed.). México: Limusa

Universidad Pedagógica Experimental Libertador UPEL. (2003). Manual de trabajos de grado de especialización y maestría y tesis doctorales. Caracas FEDUPEL 
Unesco. (1996). La Educación encierra un tesoro. Informe de la Comisión Internacional de Educación para el siglo XXI. Informe Delors. Madrid: Santillana 